\title{
The Heterodyne Instrument for the Far Infrared - An Instrument for the Herschel Space Observatory
}

\section{Timothy Finn ${ }^{1}$}

Centro Astronómico de Yebes

Yebes, Guadalajara, Spain

E-mail:t.finn@oan.es

The Heterodyne Instrument for the Far Infrared (HIFI) is a very high resolution spectrometer that will be flown on the Herschel Space Observatory (HSO) to be launched in early 2009. HIFI is a complex off-axis mirror system which utilises state of the art, superconducting, cryogenically cooled detectors capable of achieving very low noise figures and a near quantum limited sensitivity. HIFI will survey and catalogue molecular lines in a frequency range of $480 \mathrm{GHz}$ to $1.9 \mathrm{THz}$ in 7 bands with a velocity resolution of up to $0.3 \mathrm{~km} / \mathrm{s}$. It will cover a wide range of fundamental molecular lines such as $\mathrm{H}_{2} \mathrm{O}, \mathrm{O}_{2}, \mathrm{CO}, \mathrm{H}[\mathrm{I}], \mathrm{C}[\mathrm{II}]$ and Poly Aromatic Hydrocarbons (PAHs) among many others providing insights into diverse topics such as galactic and stellar evolution, solar system chemistry and may even shed light on the origins of life. The HSO will open up a largely untapped astronomical window as the earths atmosphere is essentially opaque at a majority of the frequencies covered by the HSO and will provide a wealth of scientific data to be analysed by scientist around the globe who have contributed to making the HSO a reality.

2nd MCCT-SKADS Training School Radio Astronomy: fundamentals and the new instruments

Sigüenza (Spain)

August $26^{\text {th }}-$ September $4^{\text {th }} 2008$

${ }^{1}$ Speaker 


\section{Introduction}

The Herschel Space Observatory is a space based FIR/mm wave telescope that was originally proposed in 1982 by a consortium of European Scientist and was originally called FIRST (The Far-Infrared Space Telescope), but the name was changed in 2000 to honour William Herschel the discoverer of IR radiation. Although previous space based instruments have been launched with an overlapping frequency range (e.g ISO, Spitzer, SWAS) and FIR/mm wave ground based telescopes exist (e.g JCMT, CSO, SMA) none provide the complete frequency range coverage of Herschel and its stable observing environment. It is officially part of the ESA Horizon 2000 program of cornerstone missions that will address fundamental extraterrestrial issues both within the solar system (e.g Bepicolombo, SOHO, Cluster) and further afield (e.g LISA, GAIA, XMM Newton). The Herschel Space observatory is the first of a new generation of large aperture telescopes that will allow astronomers to observe with greater sensitivity and resolution than ever before. Table 1 below outlines the main characteristics of Herschel and displays the final polished Herschel mirror at ESTEC and an artist impression of the HSO.

Table 1 : The prime charcteristics of the HSO

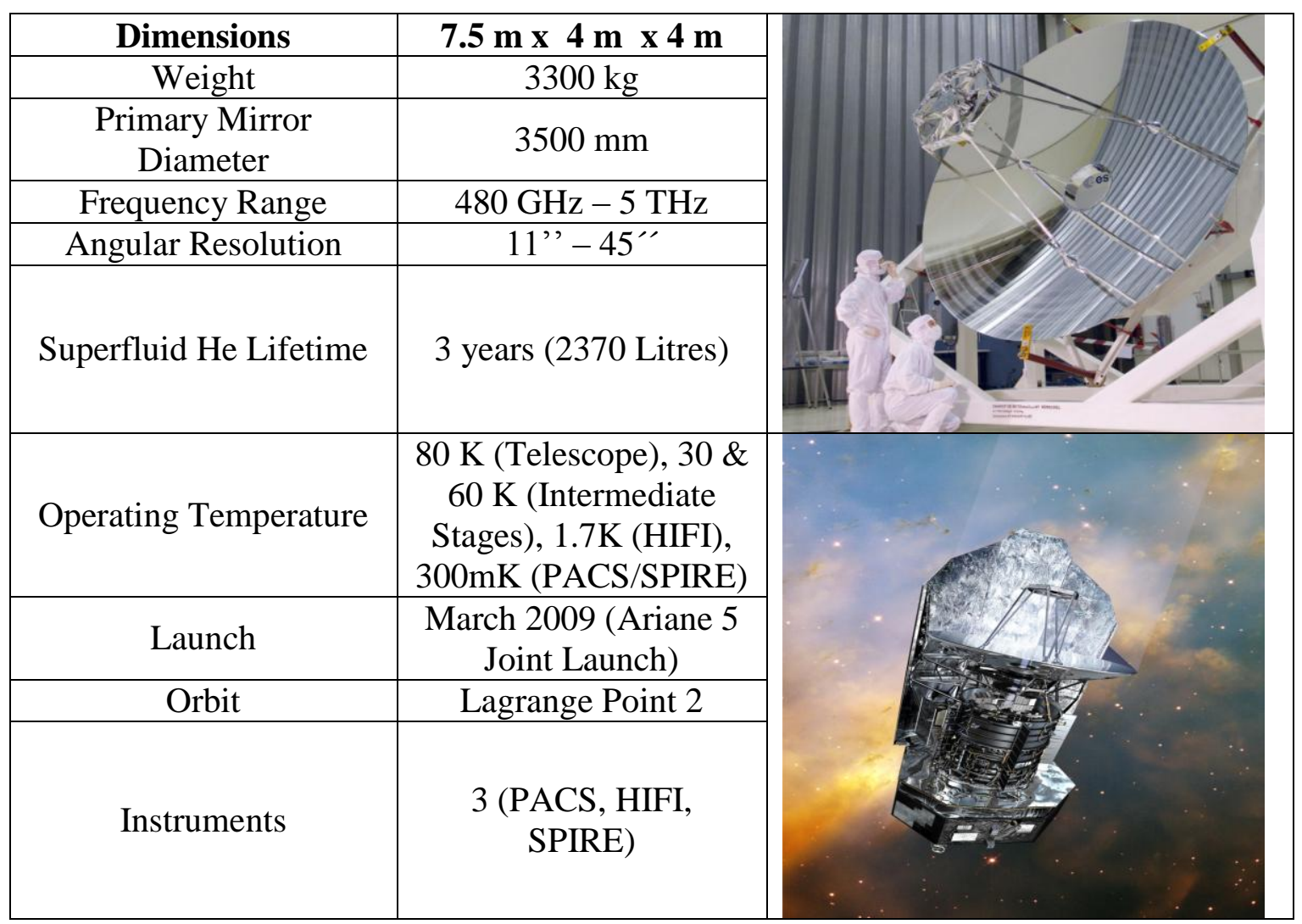




\section{Sciencentific Objectives}

The rational behind Herschel lies in the poor visibility available at sub-mm and infrared wavelenghts from the earth's surface. Even at high, dry altitudes such as the Atacama desert or Mauna Kea where water vapour content is lower than at sea level astronomers are limited to observing in limited atmospheric windows. Even with these windows they are still subject to atmospheric turbulence and IR contamination from the $300 \mathrm{~K}$ earth. Thus a space based observatory was proposed which will orbit at the L2 point, one of the five sun earth two body system points. Like all Lagrange points it is not entirely stable and thus must follow a corrective Liassajous orbit around this point which will still require course readjustments every 23 days which permits the HSO to remain in the shadow of the earth thus protecting it from the detrimental effects of the majority of the suns radiation. Figure 1 below shows the atmospheric transmission as measured at Chajnantor, Chile for a variety of prevailing water vapour levels.

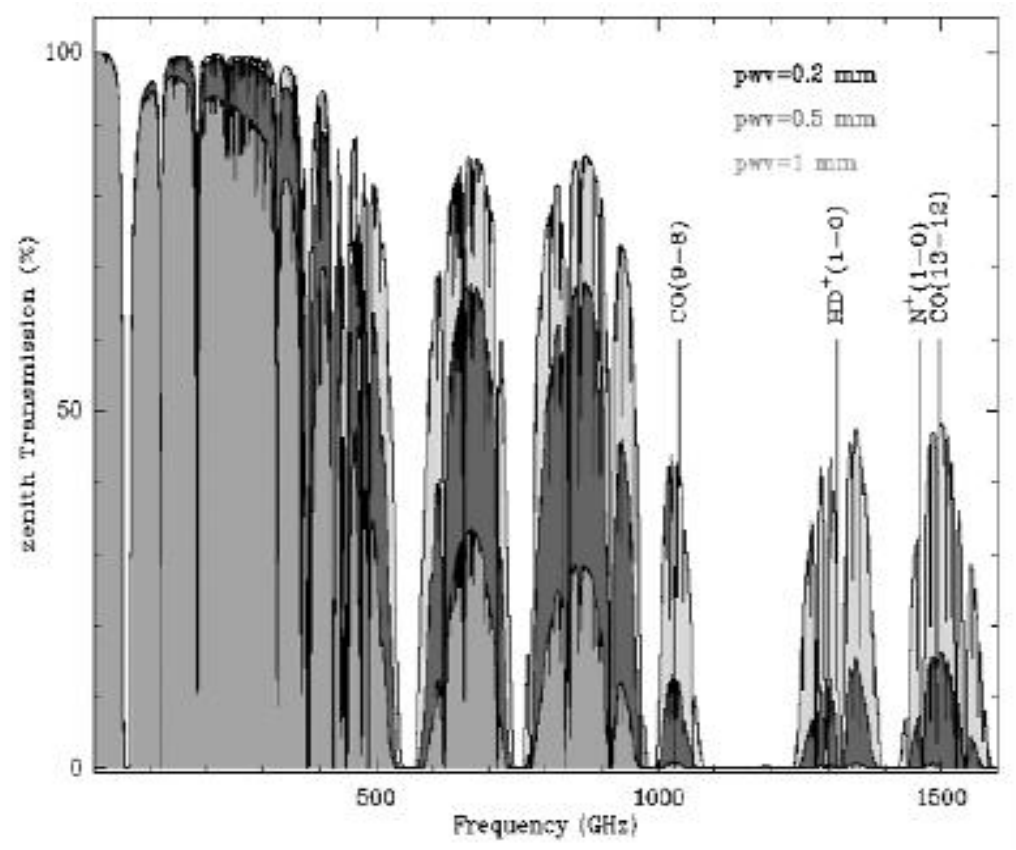

Figure 1 : Zenith atmospheric transmission at Chajnantor

Herschel has many scientific objectives the majority of which are centered on the key science projects. These have been determined by the Principal Investigators of the three instruments on board plus the scientific committee of Herschel and broadly speaking consist of:

- Observations of the cold universe (approximately $10 \mathrm{~K}-100 \mathrm{~K}$ )as it contains a large proportion of young star forming galaxies which are obscured by dust envelopes

- Observations of red-shifted radiation (Cosmology) to trace the evolution of global star formation following on from Hubble 
- Deep extragalactic broadband photometric surveys to investigate the formation and evolution of galaxy bulges and elliptical galaxies in the first third of the present age of the Universe

- Observe the brightest cooling lines of interstellar gas (e.g $\mathrm{CO}, \mathrm{H}_{2} \mathrm{O}$ ) to give important information on the physical processes in galaxies

- Studies of the physics and chemistry of the ISM

- Observe the stellar/interstellar lifecycle

- High-resolution spectroscopy of comets and the atmospheres of the cool outer planets and their satellites.

The three instruments on-board have been designed specifically to maximize the scientific return centered on the Key Program topics. In order to realize the full potential of Herschel a comprehensive technology development campaign has been undertaken to provide state of the art detection and unparalleled sensitivity. Technological challenges met include a low volume, low consumption solid state Local Oscillator (LO) that provides full frequency coverage from $480 \mathrm{GHz}$ up to $2 \mathrm{THz}$ and SuperconductingInsulator-Superconducting (SIS)/Hot Electron Bolometers (HEB) junctions with state of the art noise temperatures.

Herschel has some overlap with previous IR space missions such as Infrared Space Observatory, Spitzer, SWAS and several ground based telescopes to aid in the identification of objects and spectral lines of interest. However given its large operating band width and its location at L2 its forward scientific planning must, in many respects, be well planned out in particular by laboratory work to identify strong spectral lines complemented by sound theoretical models to predict other astronomical objects of interest. This makes Herschel particularly challenging since it must act, to a certain extent, as its own pathfinder and refine its scientific strategy during its lifetime.

\section{Payload}

The science module of Herschel consists of three instruments PACS (Photoetector Array Camera and Spectrometer), SPIRE (Spectral and Photometric Imaging Receiver) along side HIFI.

PACS is capable of both imaging photometry and spectrometry from $55-210 \mu \mathrm{m}$. It can make observations in two bands simultaneously and utilises bolometer arrays which are colled to $300 \mathrm{mK}$ and yield a point source sensitivity of $3-5 \mathrm{mJy}$. It will carry out deep multi-band photometric and spectal surveys.

SPIRE is an imaging photometer which operates in three bands centred at 250, 350 and $500 \mu \mathrm{m}$ and imaging Fourier Transform Spectrometer which provides simultaneous observation of the whole band from $194-672 \mu \mathrm{m}$. Its detectors are hexagoanl spiderweb bolometer arrays cooled to $300 \mathrm{mK}$ and which provide a sensitivity of up to 1.8 mJy. 


\subsection{HIFI}

HIFI is very high resolution heterodyne spectrometer which operates in 7 bands (14 sub-bands) from 150 - $625 \mu \mathrm{m}$ detecting the two polarisations of the incoming radiation separately. Its is capable of operating only one sub-band in one or two polarisations at a time with instantaneous bandwidth of between 2.4 and $4 \mathrm{GHz}$. Its on board detectors are superconducting devices based on SIS and HEB technologies cooled to $1.7 \mathrm{~K}$ and capable of near quantum noise level sensitivities (goal $<3 \mathrm{hf} / \mathrm{k}$ ). A key component of HIFI is the LO oscillator chain which allows HIFI to down convert high frequency astronomical signals down to microwave frequencies which can easily be managed by current electronics. The LO chain is fed by $\mathrm{W}$ band power amplifiers capable of generating up to $400 \mathrm{~mW}$. These are then frequency multiplied by planar Schottky diodes to provide a frequency stable, spectrally pure LO output for the 14 bands. The LO chain is capable of delivering up to $1 \mathrm{~mW}$ of power at $800 \mathrm{GHz}$ to the LO optics (yellow beam in figure 2).

Figure 2 below displays the optics of HIFI. The red beam is accepted from the telescope and is relayed by six Al mirrors one of which is rotatable to select bands, the calibration source or to engage beam switching on the sky, to the band splitting optics which deliver 7 beams to their respective mixer units via the diplexer which separates the two ortogonal polarisations (orange beam). A comprehensive optical analysis was undertaken to determine the efficiencies and pointing accuracies of the HIFI beams on the sky which will allow a more accurate calibration of data (goal $<10 \%$ error). After filtering and amplification the IF is further down converted to a frequency appropriate to the chosen spectrometer and each individual polarisation is delivered to the spectrometer for a particular observation. HIFI's backend consist of two wide band spectrometers based on an acousto-optical design and with a fixed resolution of 1.1 $\mathrm{MHz}$. The two high resolution spectrometers are digital auto-correlator based and capable of achieving resolutions of up to $125 \mathrm{kHz}$. This gives HIFI a potential resolving power of up to $10^{7}$ and the possibility to resolve radial velocities of down to $300 \mathrm{~m} / \mathrm{s}$.

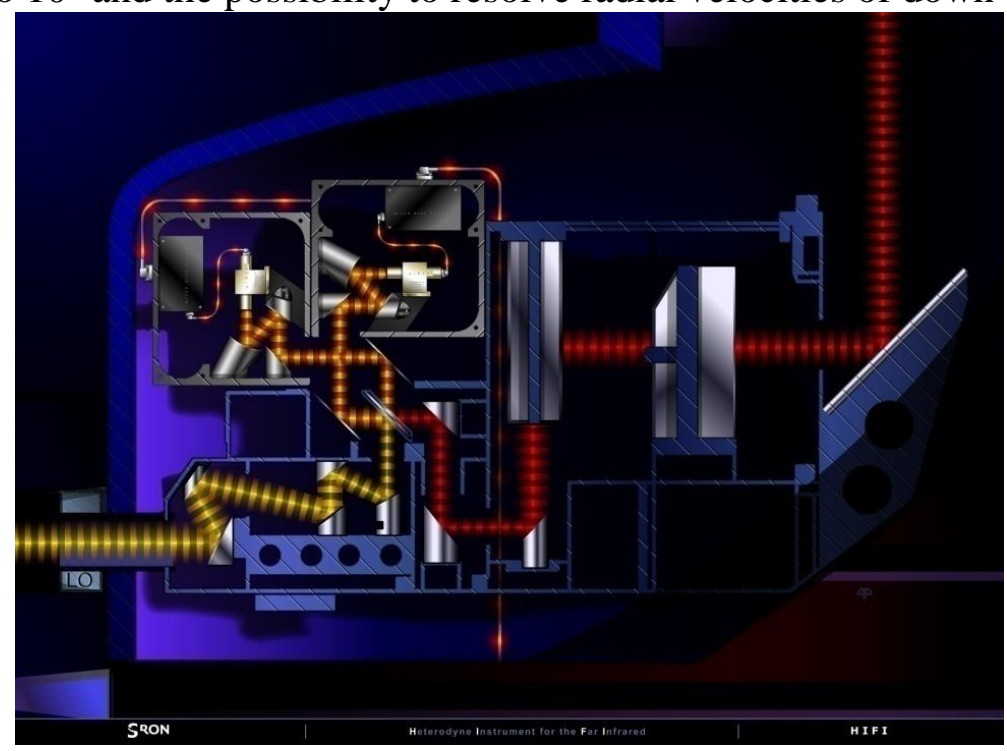

Figure 2 : The off-axis optics chain of HIFI. 


\section{Ground Segment}

Currently all three instruments are integrated on the optical bench of Herschel and have already been fully mechanically, acoustically and shock tested at ESTEC and various other Aerospace centres in Europe. The observatory is now undergoing full vacuum and radiation testing at the Large Space Simulator where all three instruments will undergo full functional tests. Depending on the outcome of these tests the observatory will be delivered to Ariane Space, Kourou early in 2009. There it will be subjected to further testing before integration in the faring of an Ariane-5ECA launcher for a dual launch along with Plank, an observatory for probing Cosmic Microwave Background radiation polarisation anisotropies.

The journey to L2 will take approximately 2 months to complete and Herschel will adopt a Lissajous orbit and where initial science demonstrations will take place before a comprehensive observation programme which will maximise returns from its foreseen 3.5 years operating lifetime. The New Norcia ground station in Australia will be responsible for up-linking and down-linking to Herschel every day while the European Space Operation Centre (ESOC) in Darmstadt, Germany is responsible for the day-today operations management of the mission. As telemetry is received by New Norcia and in turn at ESOC it is forwarded onwards to the European Space Archiving Centre in Madrid where it is released to the general community after calibration by the Instrument Control Centres (ICC) of each individual instrument on board. Figure 3 below gives an overview of the data flow within the ground segment.

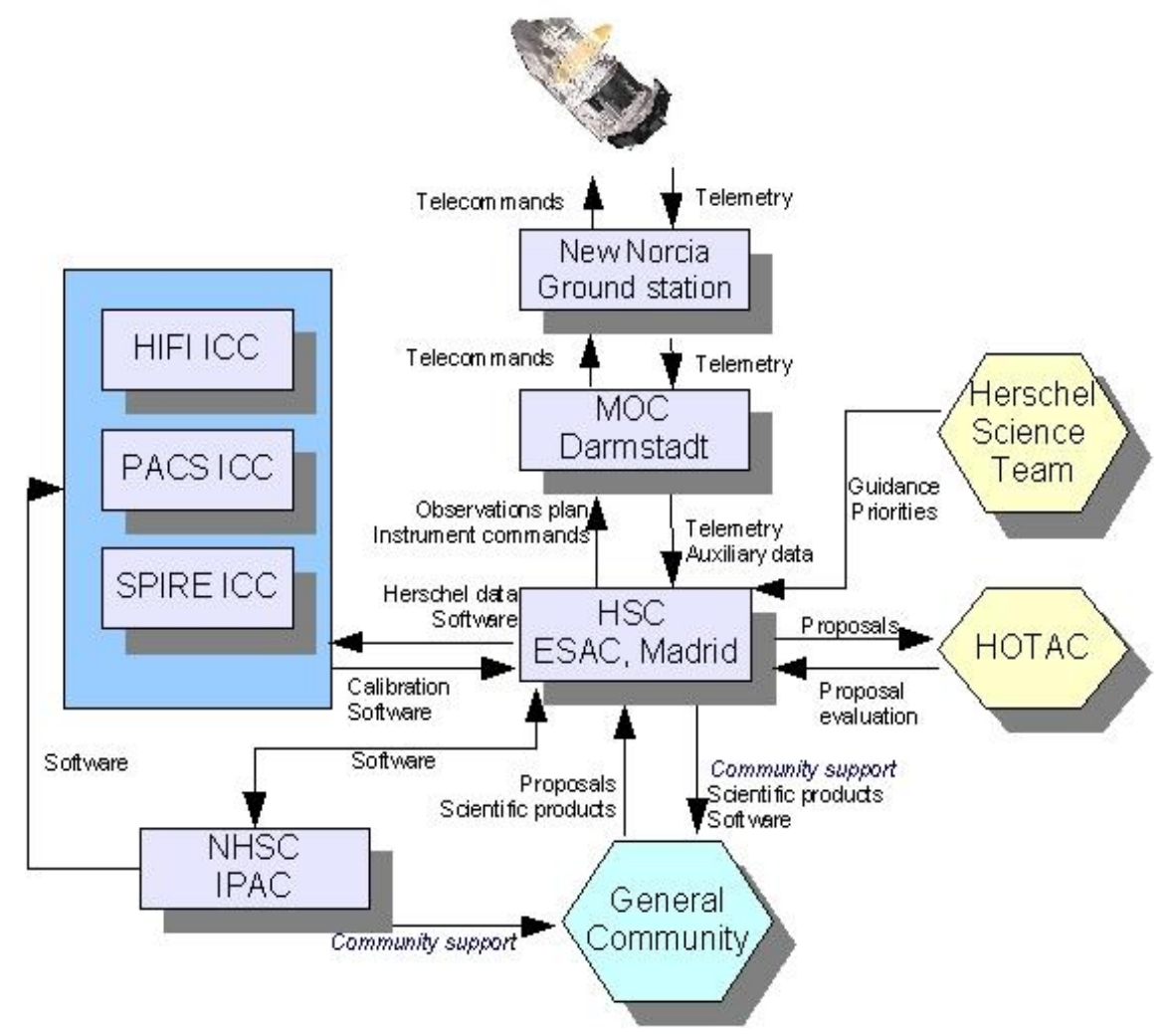

Figure 3 : The ground segment structure of Herschel 


\section{References}

[1] T de Graauw, FP Helmlich et al, "Herschel-HIFI : The Heterodyne Instrument for the Far Infrared", The Promise of the Herschel Space Observatory, ESA SP-460, pp 45-51, 2001

[2] T de Graauw, N Whyborn et al, "The Herschel-Heterodyne Instrument for the Far Infrared and pre-launch testing”, SPIE Proc 7010 : Space Telescopes and Instrumentation 2008: Optical, Infrared, and Millimeter, 2008

[3] The Herschel Science Centre, http://herschel.esac.esa.int/

[4] The HIFI website, www.sron.nl/divisions/lea/hifi/ 